\title{
Benign metastasizing leiomyoma in triple location: lungs, parametria and appendix
}

\author{
Renata Raś ${ }^{1}$, Mariusz Książek², Edyta Barnaś ${ }^{3}$, Joanna Skręt-Magierło ${ }^{1}$, Wojciech Kąziołka \\ Ludmiła Fudali², Justyna Filipowska ${ }^{5}$, Andrzej Skręt ${ }^{1}$ \\ ${ }^{1}$ Obstetrics and Gynecology Clinic, Frederick Chopin Clinical Provincial Hospital No 1, University of Rzeszow, Rzeszow, Poland \\ ${ }^{2}$ Clinical Department of Pathology, Frederick Chopin Clinical Provincial Hospital No 1, Rzeszow, Poland \\ ${ }^{3}$ Institute of Obstetrics and Emergency Medicine, Rzeszow, Poland \\ ${ }^{4}$ Department of Thoracic Surgery, Podkarpackie Center of Lung Diseases, Rzeszow, Poland \\ ${ }^{5}$ Clinical Department of Radiology, Frederick Chopin Clinical Provincial Hospital No 1, Rzeszow, Poland
}

\begin{abstract}
Benign metastasizing leiomyoma (BML) usually are situated in one organ, most often in lungs. BML patients typically have a history of uterine leiomyoma treated with hysterectomy, myomectomy or subtotal hysterectomy. The aim of the study was to present the case of a 53-year-old woman with triple location in the lungs, parametria and appendix. She had undergone a myomectomy 26 years earlier. In 2015, she was admitted to the surgical department because of abdominal pain, whereupon a cholecystectomy was performed. CT scans showed pelvic mass with pulmonary metastasis. Upon discharge the patient was referred to the Gynecology Clinic, where a laparotomy was performed. The intraoperative findings were: 1) uterus with multiple leiomyomas, 2) four tumors in the parametria, 3) tumor connected to the appendix. A subtotal hysterectomy, with a bilateral salpingo-oophorectomy, removal of the tumors from the parametria and appendectomy was performed. Pathology confirmed the diagnosis based on morphology and immunohistochemical staining (strongly positive for estrogen receptors and SMA, while Ki67 was very low, below 1\%). Upon postoperative recovery, the patient was referred to the Thoracic Surgery Department. During the thoracotomy, multiple nodes, surrounded by lung parenchyma, were revealed. Wedge resection was performed, for localized pulmonary lesions, and sent for pathological examination. The final pathological diagnosis was benign metastasizing leiomyomatosis.

In conclusion, the triple location of BML could possibly be a result of a parallel different metastasizing mechanism, although it is impossible to exclude one mechanism, which may be the cause of the metastases in three locations.
\end{abstract}

Key words: benign metastasizing leiomyoma, triple location, etiology.

\section{Introduction}

The term - benign metastasizing leiomyoma (BML) - defines an unusual coexistence of benign diseases and metastases, which are characteristic of malignant tumors. Commonly, "metastases" are found distantly from the primary location of leiomyoma, usually in the lungs [1-6], and less frequently in the heart, spine, lymph nodes and bones [7-12]. The closest locations of "metastases" of the primary lesions are in the peritoneum and bowels [7, 8].

In only three reported cases, the metastases were found in two organs i.e. the lungs and retroperitoneal space [7] or the lungs and the spine [13, 14].

It appears that the mechanism behind the phenomenon of "metastases" of leiomyoma is different from that behind malignant tumors. Historically, there are three hypotheses of BML. The first hypothesis of etiology suggests that "metastases" are the by-product of the local proliferation of smooth muscles within the involved organs. The second suggests that BML represents a low grade leiomyosarcoma. The third advocates a hematogenous dissemination of the primary lesion [9, 15-18].

The aim of the study is to present the case of three BML locations and its etiological implications.

\section{Case description}

A 53-year-old woman had undergone a myomectomy 26 years earlier. In 2015, she was admitted to the surgical department of the municipal hospital, com- 
plaining of abdominal pain, whereupon a cholecystectomy was performed, due to acute cholecystitis. During her stay in the surgical department, a pelvic mass with pulmonary metastasis was diagnosed. Chest and pelvic CT scans are shown in Fig. 1.

Upon discharge the patient was referred to the Gynecology Clinic, where a laparotomy was performed. The intraoperative findings were: 1) uterus with multiple leiomyomas, 2) four tumors in the parametria, 3) tumor connected to the appendix. A subtotal hysterectomy, with a bilateral salpingo-oophorectomy, removal of the tumors from the parametria and appendectomy was performed. The patient's postoperative course was uneventful. The pathological report revealed macroscopically: 1) the uterus with multiple fibroids, measuring from $0.5 \mathrm{~cm}$ up to $5 \mathrm{~cm}$ in maximum diameter, 2 ) in the parametria, well-circumscribed solid tumors, with the morphology of fibroids, similar to those from the uterus, measuring from $1.2 \mathrm{~cm}$ up to $9 \mathrm{~cm}$ in maximum diameter and 3) a fibroid-like nodule measuring $5 \mathrm{~cm}$ in diameter, attached to the serosal surface of the appendix. Microscopically: 1) the presence of benign leiomyomas in the uterus (Fig. 2A); no necrosis, only mild, focal atypia was noted within several of these lesions; the mitotic count was up to 4 mitosis/10 HPF; 2) the fibroid-like nodules, from the parametria, confirmed the presence of multiple, microscopically benign smooth muscle tumors, consistent with benign leiomyomas; several exhibited the presence of foci of endometriosis - so called benign adenomyomas (Fig. 2B); there was no necrosis, no marked atypia, and mitotic activity was low, less than 3 mitosis/10 HPF; the immunohistochemistry, which was performed, excluded GIST (gastrointestinal stromal tumor - CD117 negative) and nerve sheath tumors (S100 negative); the estrogen (Fig. 2C) and SMA (Fig. 2D) stains were positive, while Ki67 was very low, below 1\%; and 3) the nodule of the appendiceal location, showing morphology typical of microscopically benign smooth muscle tumors, with diffused degenerative types of changes, consistent with a benign leiomyoma; there was no necrosis, no marked atypia, and mitotic activity was low, up to 2 mitosis/ $10 \mathrm{HPF}$; the immunohistochemistry performed excluded GIST (gastrointestinal stromal tumor - CD117 negative) and nerve sheath tumors (S100 negative) and the appendix showed no pathological features.

Upon postoperative recovery, the patient was referred to the Thoracic Surgery Department. The fiberobronchoscopy, negative for any pathological findings, was followed by a thoracotomy where multiple nodes, surrounded by lung parenchyma, were revealed. Wedge resection was performed, for localized pulmonary lesions, and sent for pathological examination. After surgery, the lungs were successfully re-inflated, and on the 3rd day the patient was discharged. Macroscopic examination revealed 8 fibroid-like nodules, some with cystic changes, measuring from 7 to $27 \mathrm{~mm}$ in maximum diameter, surrounded by lung parenchyma and pleura (sub pleural locations). Microscopic examination confirmed the presence of multiple small, well-demarcated
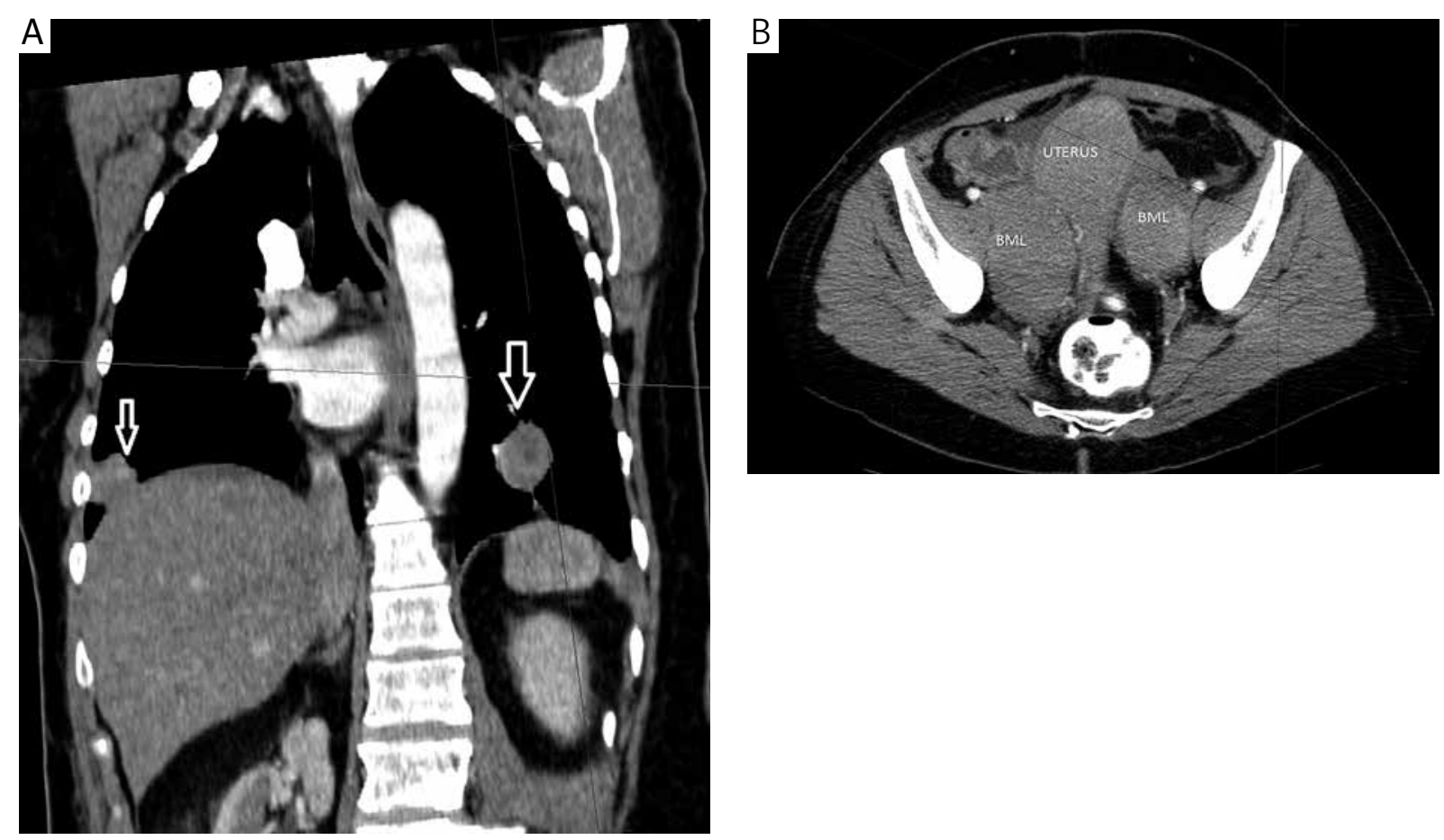

Fig. 1. CT scans: A) chest frontal scan, B) pelvic transverse scan. Arrows show lung metastases, BML show parametrial metastases 

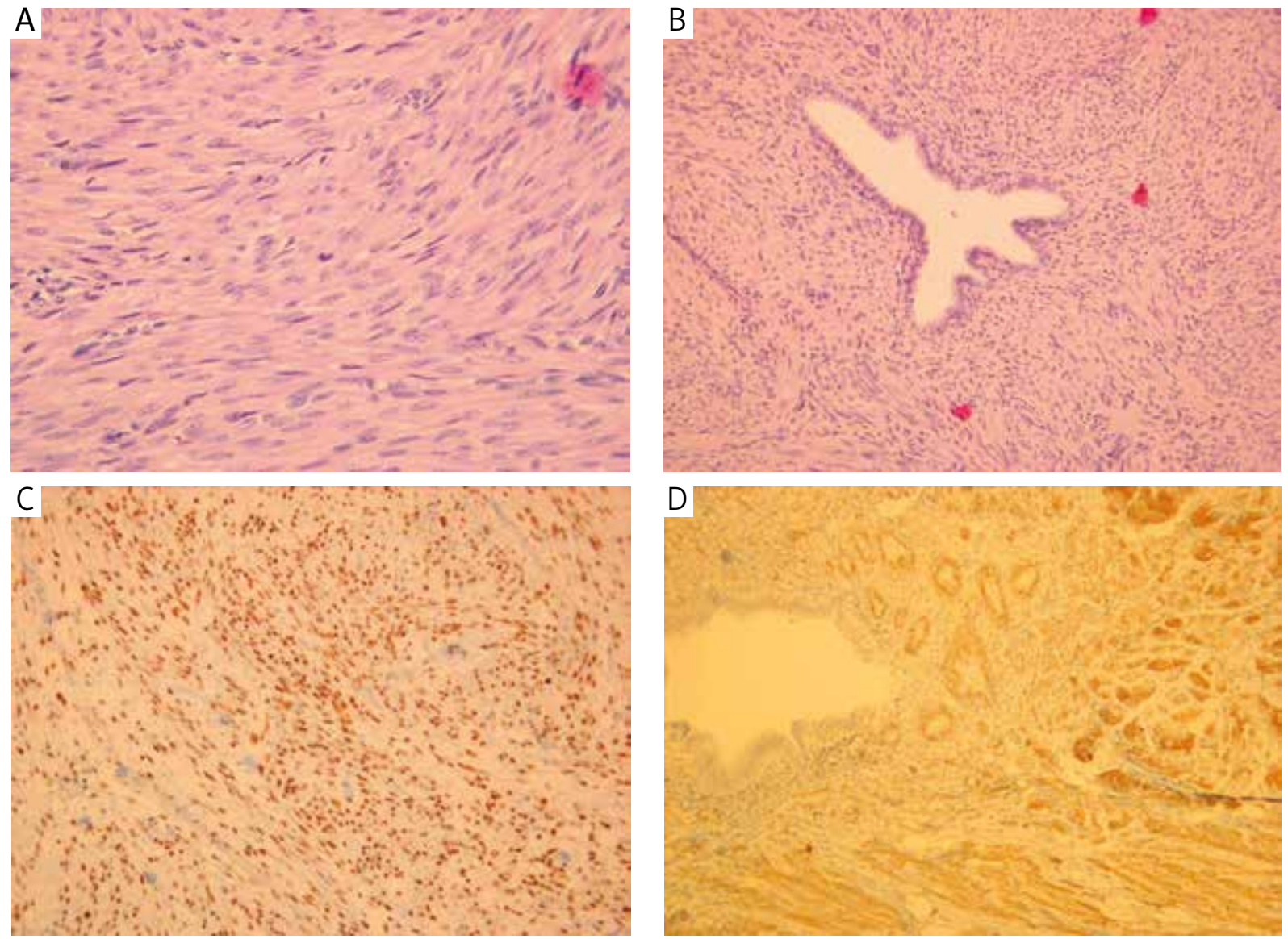

Fig. 2. Microscopic pictures of benign leiomyomas from the uterus, parametrial and appendiceal locations. A) Objective 20x, $\mathrm{H} \& \mathrm{E}$, benign leiomyoma from the uterus, sparse mitotic figure in the center (mitotic activity up to 4/10 HPF); B) Objective 20x, $\mathrm{H} \& \mathrm{E}$, benign adenomyoma (leiomyoma plus endometriosis) from the abdominal cavity, parametrial location; C) Objective 20x, IHCh positive staining for estrogen receptor, parametrial leiomyoma; D) Objective 20x, IHCh positive staining for SMA in parametrial adenomyoma

nodules consisting of bland smooth muscle cells, with no evidence of necrosis or atypia. The mitotic activity was very low: up to 1 mitotic figure/10 HPF. Areas of entrapped alveolar and bronchiolar epithelial components were seen (Fig. 3A and 3B). There were no features of intravenous leiomyomatosis. The desmin (Fig. 3B) and estrogen (Fig. 3C) stains were positive, while Ki67 was very low (Fig. 3D). The final pathological diagnosis was benign metastasizing leiomyomatosis.

\section{Discussion}

In the case we described, the myomectomy was the primary procedure - similar as in most other cases of BML. Other procedures, as described in articles, included a total or subtotal hysterectomy [9, 15-19]. There were only 3 cases described where female patients were diagnosed with $\mathrm{BML}$ without any prior gynecological surgery $[1,9,20]$. However, there was seen a coexistence of BML and uterine leiomyomas. In our case, the triple location of metastases also coexisted with the uterine leiomyomas, however the patient previously had already been treated by a surgical procedure, i.e. myomectomy.

In medical literature, the time from surgery to the appearance of metastases differs, from one year to 37 years [10, 13, 15, 21-23]. Only in two studies the time from surgery to the clinically confirmed metastasis was two and four weeks, respectively $[18,19]$. Interestingly, the shortest time to metastasis was connected with the pulmonary location, associated with previous myomectomy. However, in our case it was quite prolonged - 26 years. As stated in the introduction, the most frequent single location of $B M L$ is in the lungs [1-6]. Double locations are rare [7, 13, 14]. According to our best knowledge in literature, cases of the triple location of BML do not exist.

The presentation of the triple location of BML may have some implications with respect to the etiology of the disease. The parallel metastasis of the three locations may suggest different methods of metastases spread, for example through the bloodstream to spread to the lungs, or through the implantation/direct depos- 

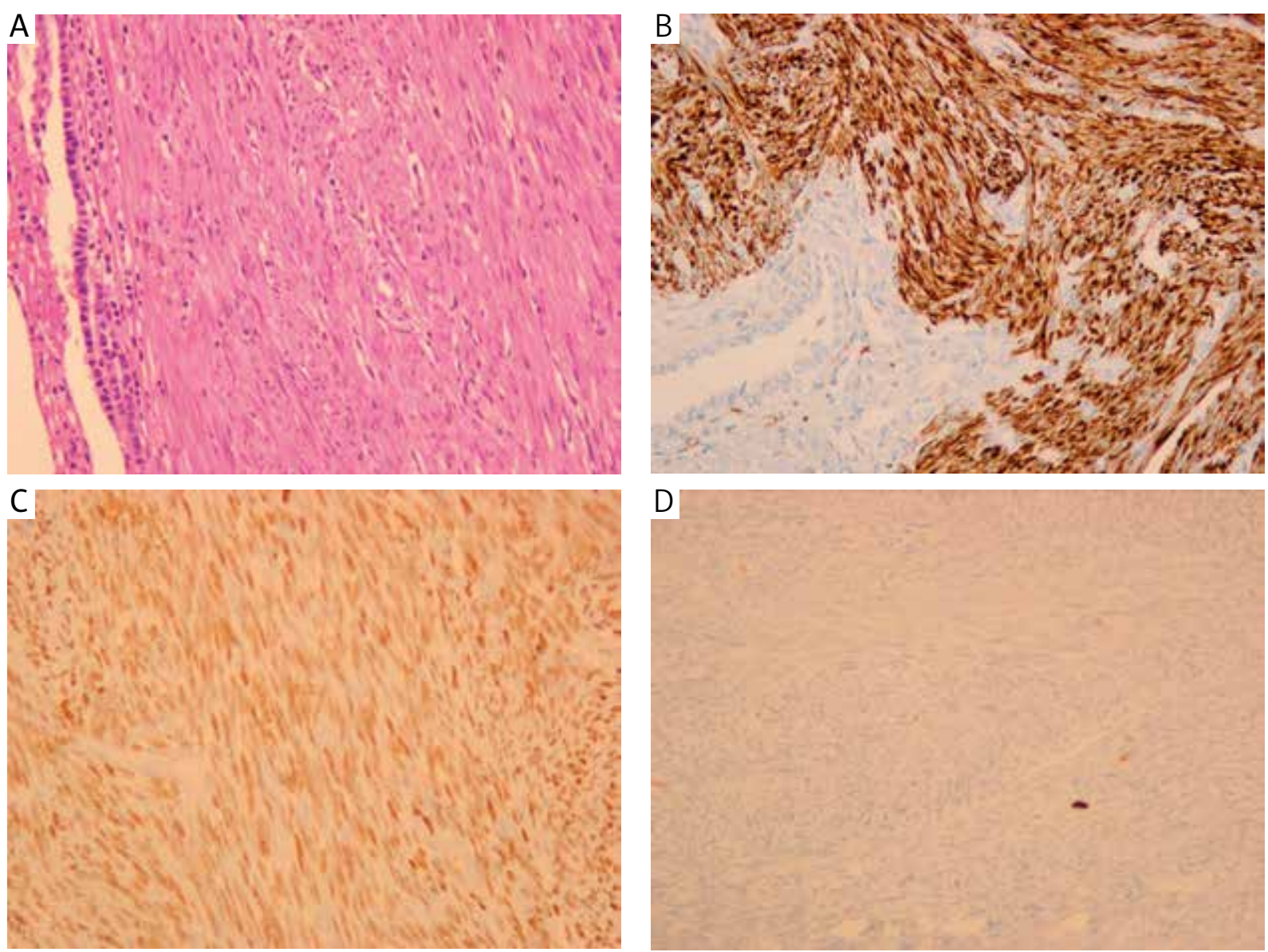

Fig. 3. Microscopic pictures of benign metastasizing leiomyomas (BML) from the lungs. A) Objective 20x, H\&E, Well-circumscribed smooth muscle tumor is present in the lung, with the features in keeping with a benign leiomyoma, on the left showing an incorporated space with entrapped alveolar and bronchiolar epithelial lining components. B) Objective 20x, Desmin positive smooth muscle cells. The left bottom corner of the picture shows an incorporated space lined with entrapped alveolar and bronchiolar epithelial components. C) Objective 20x, Estrogen positive receptors in a benign metastasizing smooth muscle nodule. D) Objective 20x, Ki 67 index very low, below $1 \%$

its, as with metastasis to parametria or the neighboring appendix. The presented case does not exclude the possibility of the formation of BML from the local proliferation of smooth muscles, native to the organs affected by BML.

Articles which describe BMLs are usually case reports, and their retrospective nature does not allow for drawing of equivocal conclusions. In the cases described, the primary and also the "metastatic" lesions had the same H\&E morphology and an identical immunohistochemistry profile. None of them showed features of marked atypia, no increased mitotic activity and no features of necrosis. Furthermore, in our case, the microscopic features of these smooth muscle lesions, examined from the uterus, parametria, periappendiceal location and the lungs, were practically identical in both their morphology and immunohistochemistry profile. Several lesions originating from the uterus and peritoneum showed some foci of endometriosis. The pul- monary lesions showed incorporated spaces, with entrapped alveolar and bronchiolar epithelial components.

In the future, quite possibly, new immunohistochemistry methods will permit us to differentiate between the primary lesion and metastases.

Descriptions of BML cases come most often from the thoracic surgery departments, intensive care units, neurosurgery, and oncology departments. Gynecology departments very rarely view such cases. There are only 4 cases of BML described in Poland [3, 21, 23, 24].

\section{Conclusion}

In conclusion, the triple location of BML could possibly be a result of a parallel different metastasizing mechanism, although it is impossible to exclude one mechanism, which may be the cause of the metastases in the three locations. 


\section{Disclosure}

\section{Authors report no conflict of interest.}

\section{References}

1. Taveira-DaSilva AM, Alford CE, Levens ED, et al. Favorable response to antigonadal therapy for a benign metastasizing leiomyoma. Obstet Gynecol $2012 ; 119(2$ Pt 2): 438-442.

2. Taftaf R, Starnes S, Wang J, et al. Benign metastasizing leiomyoma: a rare type of lung metastases-two case reports and review of the literature. Case Rep Oncol Med 2014; 2014: 842801.

3. Radzikowska E, Szczepulska-Wójcik E, Langfort R, et al. Benign pulmonary metastasizing leiomyoma uteri. Case report and review of literature. Pneumonol Alergol Pol 2012; 80: 560-564.

4. Wei WT, Chen PC. Benign metastasizing leiomyoma of the lung: A case report and literature review. Oncol Lett 2015; 10: 307-312.

5. Goto T, Maeshima A, Akanabe K, et al. Benign metastasizing leiomyoma of the lung. Ann Thorac Cardiovasc Surg 2012; 18: 121-124.

6. Ogawa M, Hara M, Ozawa Y, et al. Benign metastasizing leiomyoma of the lung with malignant transformation mimicking mediastinal tumor. Clin Imaging 2011; 35: 401-404.

7. Ahmad SZ, Anupama R, Vijaykumar DK. Benign metastasizing leiomyoma - case report and review of literature. Eur J Obstet Gynecol Reprod Biol 2011; 159: 240-241.

8. Mizuno M, Nawa A, Nakanishi T, et al. Clinical benefit of endocrine therapy for benign metastasizing leiomyoma. Int J Clin Oncol 2011; 16: 587-591.

9. Del Real-Romo ZJ, Montero-Cantú C, Villegas-Cabello O, et al. Incidental benign metastasizing leiomyoma in a patient with bone sarcoma: a case report. Case Rep Surg 2014; 2014: 439061.

10. Consamus EN, Reardon MJ, Ayala AG, et al. Metastasizing leiomyoma to heart. Methodist Debakey Cardiovasc J 2014; 10: 251-254.

11. Hur JW, Lee S, Lee JB, et al. What are MRI findings of Spine Benign Metastasizing Leiomyoma? Case report with literature review. Eur Spine J 2015; 24 Suppl 4: S600-5.

12. Duncan SJ, Kadaria D. A Woman with a lung mass and multiple pulmonary nodules. Am J Case Rep 2015; 16: 367-370.

13. Berti AF, Santillan A, Velasquez LA. Benign metastasizing leiomyoma of the cervical spine 31 years after uterine leiomyoma resection. J Clin Neurosci 2015; 22: 1491-1492.

14. Wang LX, Lv FZ, Ma X, et al. Multifocal osteolytic lesions within lumbar spine in a middle-aged Chinese woman: a benign metastasizing leiomyoma? Spine (Phila Pa 1976) 2012; 37: E259-63.

15. Ma H, Cao J. Benign pulmonary metastasizing leiomyoma of the uterus: A case report. Oncol Lett 2015; 9: 1347-1350.

16. Yoon G, Kim TJ, Sung CO, et al. Benign metastasizing leiomyoma with multiple lymph node metastasis: a case report. Cancer Res Treat 2011; 43: 131-133

17. Lim SY, Park JC, Bae JG, et al. Pulmonary and retroperitoneal benign metastasizing leiomyoma. Clin Exp Reprod Med 2011; 38: 174-177.

18. Yu R, Ferri M. An unusual cause of pulmonary nodules in the emergency department. Case Rep Emerg Med 2015; 2015: 278020.

19. Chen S, Liu RM, Li T. Pulmonary benign metastasizing leiomyoma: a case report and literature review. J Thorac Dis 2014; 6: E92-E98.

20. Rege AS, Snyder JA, Scott WJ. Benign metastasizing leiomyoma: a rare cause of multiple pulmonary nodules. Ann Thorac Surg 2012; 93: e149e151.

21. Kołaczyk K, Chamier-Ciemińska K, Walecka A, et al. Pulmonary benign metastasizing leiomyoma from the uterine leiomyoma: a case report. Pol J Radiol 2015; 80: 107-110.

22. Cadieux-Simard ML, Hennessey H, Small D, et al. latrogenic exacerbation of pulmonary arteriovenous malformation in a patient with benign metastasizing leiomyoma. Respirol Case Rep 2014; 2: 15-17.

23. Jeon HW, Choi SH, Sung SW, et al. Pulmonary benign metastasizing leiomyoma: report of three cases. World J Surg Oncol 2013; 11: 281.

24. Jęda A, Karoń J, Rak M. The nodule of the small intestine - a rare case of benign metastasizing leiomyoma. Ginekol Pol 2009; 80: 716-719. 